\title{
TURBULENCE DRIVEN BY FARADAY SURFACE WAVES*
}

\author{
MICHAEL SHATS \\ Research School of Physics and Engineering, The Australian National University, \\ Canberra, ACT 0200, Australia \\ Michael.Shats@anu.edu.au \\ NICOLAS FRANCOIS \\ Research School of Physics and Engineering, The Australian National University, \\ Canberra, ACT 0200, Australia \\ Nicolas.Francois@anu.edu.au \\ HUA XIA \\ Research School of Physics and Engineering, The Australian National University, \\ Canberra, ACT 0200, Australia \\ Hua.Xia@anu.edu.au \\ HORST PUNZMANN \\ Research School of Physics and Engineering, The Australian National University, \\ Canberra, ACT 0200, Australia \\ Horst.Punzmann@anu.edu.au
}

\begin{abstract}
We report experimental results which show that the particle motion on the surface perturbed by Faraday waves is similar to the fluid motion in 2D turbulence. It supports the inverse energy cascade or the spectral energy transfer from smaller to larger scales. The vertical acceleration ranges from the Faraday instability threshold up to the droplet nucleation threshold where the ripples are a couple of millimeters high. Such a configuration rules out any $2 \mathrm{D}$ assumption on the fluid motion. The motion of floaters on the surface of the Faraday waves is essentially three dimensional but its horizontal component shows unexpected analogy with two-dimensional turbulence. The presence of the inverse cascade is detected by measuring frequency spectra of the Lagrangian velocity and confirmed by computing the third moment of the horizontal Eulerian velocity fluctuations. This is a robust phenomenon observed in deep water in a broad range of flow energies and wavelengths. The emergence of such a phenomenology in Faraday waves broadens the applicability of features common to $2 \mathrm{D}$ turbulent flows to the context of surface wave phenomena which is prevalent in many systems.
\end{abstract}

Keywords: 2D turbulence; Faraday waves; spectral condensate.

\footnotetext{
* This is an Open Access article published by World Scientific Publishing Company. It is distributed under the terms of the Creative Commons Attribution 3.0 (CC-BY) License. Further distribution of this work is permitted, provided the original work is properly cited.
} 


\section{Introduction}

In turbulence energy is transferred across length scales. In the absence of dissipation, energy flux in the wave number space is conserved. In 3D turbulence this flux is directed from a large scale, at which turbulence is forced, to small scales, where it is dissipated by viscosity ${ }^{1}$. In $2 \mathrm{D}$ turbulence, energy cascades from smaller scales towards larger scales in the process known as the inverse energy cascade ${ }^{2}$. First experiments on turbulence in thin fluid layers designed to model 2D turbulence confirmed basic expectations of Kraichnan's theory, namely the existence of the inverse energy cascade and the generation of the Kolmogorov $k^{-5 / 3}$ spectrum $^{3,4}$. Later it has been realized that these are not restricted to thin layers, but are rather universal properties of flows in thick layers ${ }^{5}$. The presence of coherent structures, either self-generated via spectral condensation in bounded flows, or externally imposed, enforces two-dimensionality in the flow securing the inverse energy cascade and the energy transfer towards large scales ${ }^{6}$. Recently such a scenario has been reported from the aircraft measurements in the hurricane boundary layer ${ }^{7}$. It has been shown that at heights above $150 \mathrm{~m}$ the sign of the third-order structure function is positive, indicating the inverse energy cascade and the possibility that a hurricane may actually gain energy from small scales.

Even more recently, it has been discovered that motion of fluid parcels on the surface of the Faraday ripples represents an excellent model of 2D turbulence ${ }^{8,9}$. Such turbulence exhibits the inverse energy cascade, and if bounded, can generate large scale coherent structures due to spectral condensation on the box size. Since motion of fluid particles on the indulating surface is $3 \mathrm{D}$, Faraday wave driven turbulence represents yet another interesting example of the 3D flow showing properties of 2D turbulence. Understanding such flows is important from both fundamental and practical perspectives. The main feature of $2 \mathrm{D}$ turbulence, the inverse energy cascade, is the mechanism responsible for the generation of coherent structures in bounded systems, or spectral condensates ${ }^{10}$. It has recently been found in numerical simulations that the inverse energy cascade might be a general principle existing in both $2 \mathrm{D}$ and $3 \mathrm{D}$ turbulent flows ${ }^{11}$. It is thus important to understand the conditions under which random 3D fluid motion can lead to the generation of the spectral energy transfer from small to large scales.

In this paper we describe experimental study of motion of floating particles on the surface of the Faraday ripples.

Faraday waves are perturbations of the interface between two fluids (for example, water and air) subjected to vertical vibration. They have been studied for several hundred years producing a wealth of physics on pattern formation ${ }^{12-15}$, turbulence ${ }^{8}$, wave turbulence (e.g. Ref.16-18), instabilities and solitons ${ }^{19-23}$, physics of bouncing orbiting droplets (walkers) ${ }^{24}$, extreme wave events, or rogue waves ${ }^{25}$, and others. This object, whose systematic studies have been pioneered by M. Faraday ${ }^{26}$, still remains not fully understood. Moreover, new experimental capabilities reveal an even richer spectrum of effects in Faraday surface ripples.

The paper is organized as follows. Section 2 describes parametric wave excitation, nonlinear processes leading to the development of complex Faraday ripples, and 
describes experimental setup. Section 3 describes motion of floating particles on the surface of Faraday waves, spectra of surface elevation and Eulerian statistics of the horizontal motion of floaters, including Kolmogorov spectra and measurements of the energy flux in the energy cascade range. Section 4 summarizes the results and outlines future studies of the Faraday wave turbulence.

\section{Turbulence Generation by Faraday Waves}

\subsection{Parametric wave excitation}

Faraday pioneered experimental studies of the liquid surface ripple over 180 years ago by performing experiments with vertically vibrated containers ${ }^{26}$. When a liquid is vibrated as a whole at the frequency $\omega_{0}$, a standing wave at the frequency of the first subharmonic $\omega_{1}=\omega_{0} / 2$ is excited above some critical level of acceleration. This process can be described as a decay of the infinitely long wave $\left(\omega_{0}, \vec{k}_{0}\right)$ into two oppositely propagating subharmonic waves:

$$
\begin{gathered}
\omega_{0}=\omega_{1}+\omega_{2}=2 \omega_{1} \\
\vec{k}_{0}=\vec{k}_{1}+\vec{k}_{2} \approx 0,
\end{gathered}
$$

where $\vec{k}_{1}=-\vec{k}_{2}$, and

$$
\omega_{1}=\omega_{2}=\left(g k+\alpha k_{1}^{3} / \rho\right)^{1 / 2} .
$$

Here $g$ is the acceleration of gravity, $\alpha$ is the surface tension and $\rho$ is the fluid density. Such decay leads to the generation of waves at the frequency $\omega_{1}=\omega_{2}=\omega_{0} / 2$ often referred to as Faraday waves.

Apart from the decay of the $\vec{k}_{0}=0$ wave, three wave interactions are severely restricted for capillary waves and they are forbidden for gravity waves. For capillary waves simultaneous satisfaction of energy and momentum conservation,

$$
\omega\left(\vec{k}_{1}+\vec{k}_{2}\right)=\omega\left(\vec{k}_{1}\right)+\omega\left(\vec{k}_{2}\right)
$$

is only possible for the wave triads with disparate wave vectors, for example, $k_{0}<<k_{1}, k_{2}$, and for the angles between interacting wave vectors in the range between $75^{\circ}$ and $82^{\circ}$ (see e.g. Ref. [27]).

This means, that contrary to common belief that capillary wave dispersion universally satisfies matching rules (4), this cannot be taken for granted in the systems characterized by discrete frequency and wave number spectra. This is important since the majority of experiments on parametrically excited waves driven by monochromatic excitation show discrete frequency spectra dominated by the first subharmonic and a large number of its harmonics. In this case the condition (4) is not satisfied, and three-wave interactions are forbidden. This means that the quadratic nonlinearity plays no role in the nonlinear evolution of parametrically excited surface waves. 


\subsection{Modulation instability and formation of Faraday patterns}

Four-wave interactions, however, are allowed for surface waves. Moreover, both gravity and capillary waves are unstable to small amplitude modulations. This modulation instability, for gravity waves known as Benjamin-Feir instability ${ }^{28}$ develops when the Lighthill criterion is satisfied:

$$
\left(\partial \omega / \partial|a|^{2}\right)\left(\partial^{2} \omega_{k} / \partial k^{2}\right)<0
$$

This criterion is satisfied for both branches of surface waves since nonlinear frequency corrections have different signs: $\omega=\omega_{k}\left[1+(k a)^{2} / 2\right]$ for gravity waves, and $\partial \omega_{k} / \partial k=(k T / \rho)^{1 / 2}\left[1-(k a)^{2} / 16\right]^{-1 / 4}$ for capillary waves ${ }^{29}$. Though $\left(\partial \omega / \partial|a|^{2}\right)\left(\partial^{2} \omega_{k} / \partial k^{2}\right)<0$ is negative for gravity waves and positive for capillary waves, the Lighthill criterion (5) is satisfied for both.

Modulation instability of capillary waves was identified in Refs. 30 and 31. Figure 1 illustrates the dynamics of the wave excitation captured using fast video camera. Initially, $0.125 \mathrm{~s}$ after the forcing is switched on, Fig. 1(a), the wave fronts of the excited wave are concentric circles, reflecting the shape of the container wall. However very soon these wave fronts become azimuthally modulated, Fig. 1(b), and after about $0.6 \mathrm{~s}$ the wave field appears to be severely disordered, as in Fig. 1(c). Similar evolutions are observed for all excitation frequencies.

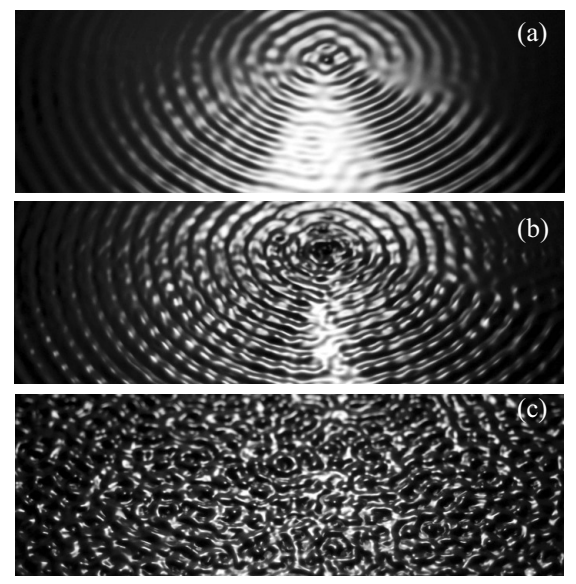

Fig. 1. Snapshots of the wave field evolution during the startup: (a) $t=0.125 \mathrm{~s}$, (b) $t=0.25 \mathrm{~s}$, (c) $t=0.625 \mathrm{~s}$. Surface ripple is excited parametrically at $f_{0}=250 \mathrm{~Hz}$.

\subsection{Experimental setup and flow visualization}

Experiments are carried out in a circular container (178 $\mathrm{mm}$ diameter, $30 \mathrm{~mm}$ deep) as shown in Figure 2. The container is filled with a liquid whose depth is larger than the wavelength of the perturbations at the surface. The monochromatic forcing is varied in the range of frequencies $f_{0}=30-60 \mathrm{~Hz}$ which results in Faraday wavelengths $\lambda$ in the 
range $\lambda=8-18 \mathrm{~mm}$. The dominant frequency of the excited surface ripples is at the first subharmonic of the excitation frequency, $f=f_{0} / 2$. We define the supercriticality as $\alpha=\left(a-a_{t h}\right) / a_{t h}$, where $a$ is the amplitude of the vertical acceleration imposed by the shaker and $a_{t h}$ is the threshold for the parametric generation of Faraday waves.

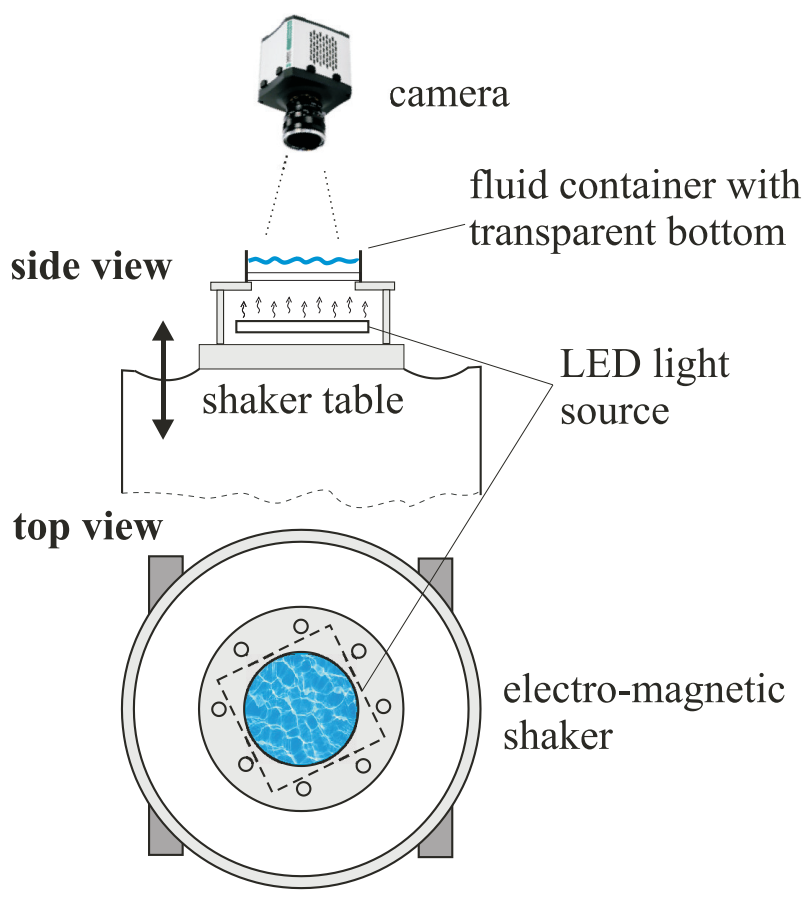

Fig. 2. Experimental setup for the surface wave driven turbulence.

A diffusing light imaging technique is used to visualize simultaneously the surface ripples and floating tracer particles, as seen in Fig. 3(a). A $2 \%$ milk solution in water provides sufficient contrast for the parametrically excited waves to be observed and allows high resolution movies $(800 \times 800$ pixel) to be recorded in the range of speeds $80-$ 120 fps using the Andor Neo sCMOS camera. Black floating particles are added on the fluid surface to visualize the horizontal motion of the liquid. Particles are made of carbon glass and have been plasma treated to reduce their intrinsic hydrophobicity. The use of surfactant and plasma treatment ensures that particles do not aggregate at the surface. This is illustrated in Fig. 3(a) with particles homogeneously distributed at the surface of the wave field. 

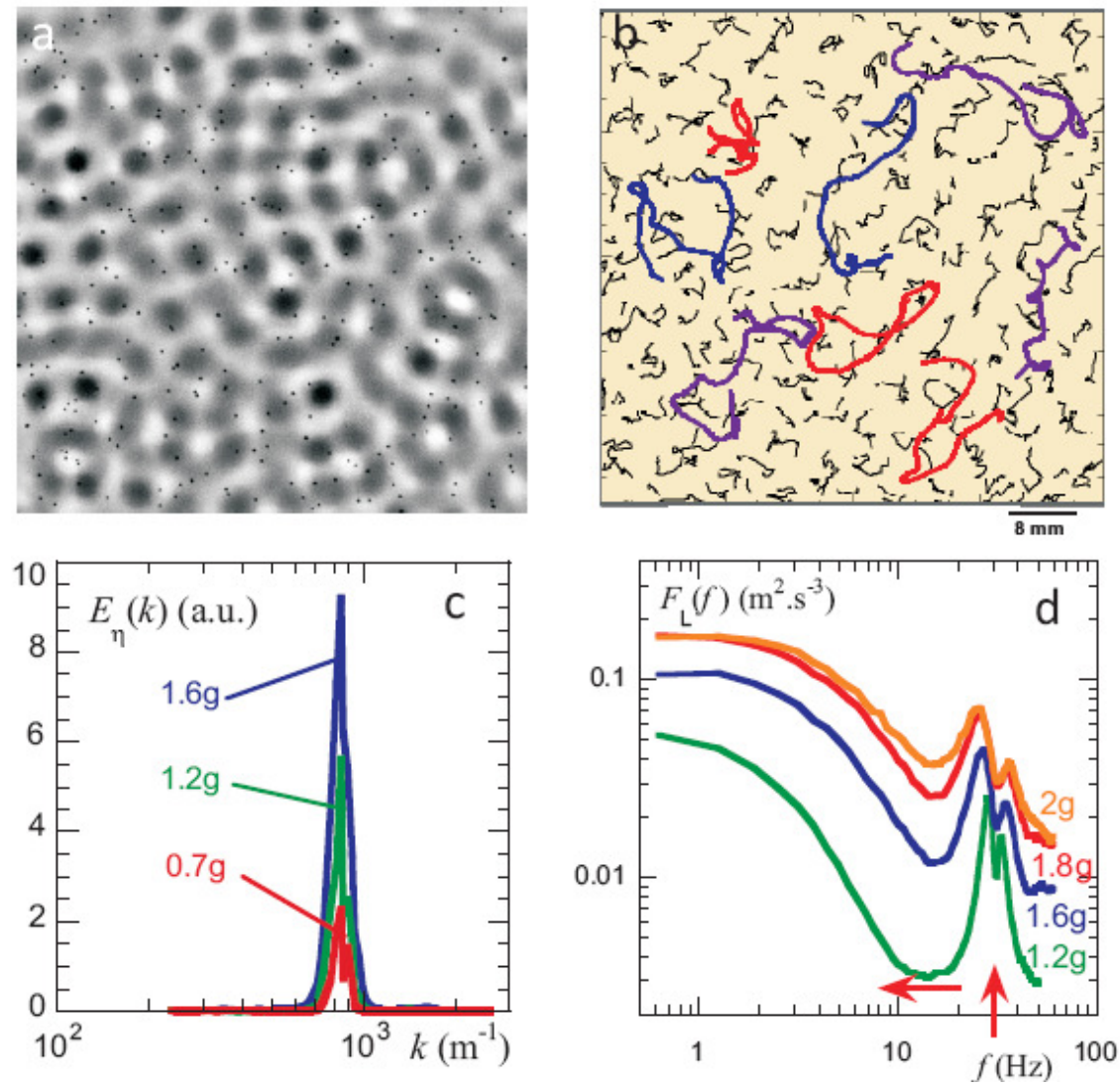

Fig. 3. (a) Diffusive light image of the fluid surface elevation including floating particles. (b) Trajectories of oscillons (black) having a phase maximum, and trajectories of 8 particles (colored) tracked for $2 \mathrm{~s}$ ( 200 periods of the shaker). (c) Wave number spectra of the surface elevation at different vertical accelerations $(a=0.7 \mathrm{~g}, 1.2$ $\mathrm{g}$, and $1.6 \mathrm{~g}$ ) and $f_{0}=60 \mathrm{~Hz}$. (d) Frequency power spectra of the Lagrangian velocity (averaged over $\sim 1000$ trajectories) for different vertical accelerations at $f_{0}=60 \mathrm{~Hz}$.

It has recently been shown that the Faraday wave properties are consistent with those of an ensemble of bounded oscillating solitons, or oscillons ${ }^{23}$. Figure 3(b) shows examples of the oscillons' trajectories to be compared with a few particle tracks measured using the same tracking code. The horizontal motion of oscillons is random in time but confined to a disordered lattice as seen from the black trajectories shown in Fig. 3(b) (see Ref. 32). The particles also wander erratically, but their trajectories show substantially larger excursions in comparison to oscillons. A particle usually visits several oscillon sites which randomize its trajectory. Although the particle and oscillons trajectories are characterized by completely different length scale, the oscillon mobility is an essential feature of Faraday flows. In the case of a perfect oscillonic crystal, in which the relative position of the nodes is frozen, the horizontal mobility of particles is zero, showing that 
the horizontal particle transport is strongly correlated to the oscillon motion. Such crystals are obtained by adding minute amounts of bovine serum to water ${ }^{23}$.

Though the oscillon lattice in Fig. 3(a) seems disordered, the wave number spectrum of the ripples is very narrow, as seen in Fig. 3(c). This is consistent with the fact that oscillon motion is restricted in space to about half of the lattice characteristic wavelength $^{32}$. To generate $2 \mathrm{D}$ turbulence one needs to inject energy into the horizontal fluid motion at some intermediate range of scales in a localized wave number domain. The horizontal mobility of oscillons is the manifestation of a momentum transfer along the water surface; this transfer is restricted to a narrow $k$-domain.

Fig. 3(d) shows frequency power spectra of velocity of particles moving along their Lagrangian trajectories. The spectra show peaks close to the Faraday frequency $(30 \mathrm{~Hz}$, the first subharmonic of the excitation frequency), and a broad low-frequency band whose energy grows with the increase in vertical acceleration. Since energy is injected at $f=30 \mathrm{~Hz}$, the growth and the broadening of the low-frequency band indicates a nonlinear energy transfer to slower temporal (larger spatial) scales. This transfer, as will be shown below, is due to the inverse energy cascade.

\section{2D Turbulence Characteristics of the Floaters Motion}

Fig. 4(a) shows many trajectories of particles on a densely seeded fluid surface, the presence of multiple length scales in this Faraday flow is visible. In the following, Particle Image Velocimetry (PIV) is used to characterize the Eulerian velocity field. The movies are recorded with a spatial resolution of $100 \mu \mathrm{m}$ and the frame rate is twice the frequency of the shaker $f_{0}$. The field of view is $8 \mathrm{~cm}$ by $8 \mathrm{~cm}$. The PIV interrogation window is a square with a $2.6 \mathrm{~mm}$ side. The energy spectra are averaged over 2000 snapshots of the velocity field. The wave number of the energy spectra is rescaled according to the notion of eddy length scale $l_{\text {eddy }}$ commonly used in turbulence ${ }^{1}$ such that $k=2 \pi / l_{\text {eddy }}$ and $l_{\text {eddy }}=\lambda_{\text {fourier }} / 2$ where $\lambda_{\text {fourier }}$ is the wavelength used in the Fourier transform.

Fig. 4(b) shows wave number spectra of the horizontal kinetic energy measured at $f_{0}=60 \mathrm{~Hz}$ at three vertical acceleration levels, $a=0.7 \mathrm{~g}, 1.2 \mathrm{~g}$, and $1.6 \mathrm{~g}$, corresponding to supercriticalities in the range $\alpha=(0.17-1.7) a_{t h}=0.6 \mathrm{~g}$ at $\left.60 \mathrm{~Hz}\right)$. The spectrum is close to the Kolmogorov-Kraichnan theory power law of $E_{v}(k) \propto k^{-5 / 3}$ at wave numbers $k \leq 1500 \mathrm{~m}^{-1}$. At higher wave numbers, $k \geq 1500 \mathrm{~m}^{-1}$, spectra are somewhat steeper than expected from the direct enstrophy cascade fit of $k^{-3}$, probably due to higher damping at large wave numbers. If we define the turbulence forcing wave number from the position of the kink on the turbulence spectrum, e.g. $k \approx 1500 \mathrm{~m}^{-1}$ at $60 \mathrm{~Hz}$, Fig. 4(b), it appears that the turbulence forcing wave number is roughly twice that of the surface elevation wave number, Fig. 3(c). The energy injection scale for the horizontal particle transport is thus related to the oscillon size which is about half of a period of the Faraday wave. 

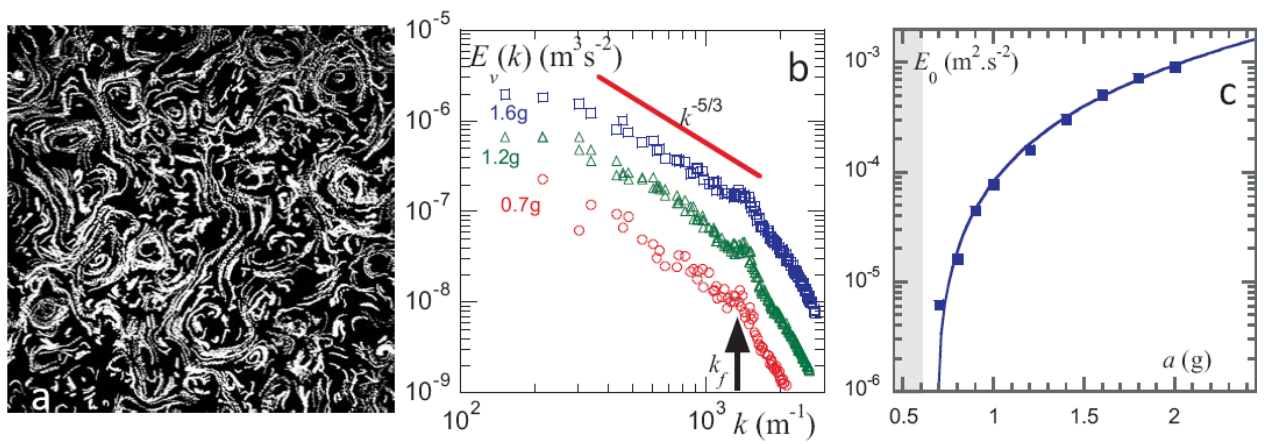

Fig. 4. (a) Particle trajectories followed over 5 periods of the shaker oscillations at $a=1.6 \mathrm{~g}$ and $f_{0}=60 \mathrm{~Hz}$ in a $8 \times 8 \mathrm{~cm}^{2}$ field of view. (b) Wave number spectra of the horizontal kinetic energy at different levels of the vertical acceleration ( $a=0.7 \mathrm{~g}, 1.2 \mathrm{~g}$, and $1.6 \mathrm{~g}$ ) and $f_{0}=60 \mathrm{~Hz}$. (c) Kinetic energy of the flow $E_{0}$ versus vertical acceleration $a$. Grey bar indicates the threshold of parametric instability.

The kinetic energy of the horizontal flow can be estimated as $E_{0}=\int_{k_{l o w}}^{k_{f}} E_{v}(k) d k$. Here, $k_{\text {low }}$ is the lowest wave number determined by the field of view. In Fig. $4 \mathrm{C}, E_{0}$ is shown as a function of the vertical acceleration $a$. By changing the vertical acceleration from $0.7 \mathrm{~g}$ to $2.4 \mathrm{~g}$, we can vary the kinetic energy in the flow by over two orders of magnitude.

Similar measurements were performed at several frequencies of the vertical vibrations: $30 \mathrm{~Hz}, 45 \mathrm{~Hz}, 60 \mathrm{~Hz}$, Fig. 5a. A $E_{v}(k) \propto k^{-5 / 3}$ power law is consistently observed in the energy spectra at lower wave numbers. A distinct kink is present in all spectra, and it is associated with the forcing wave number. The turbulence forcing wave number $k_{f}$ decreases with the decrease in frequency, in accordance with the capillarygravity wave dispersion relation. A $k^{-5 / 3}$ range in the horizontal velocity spectra is consistent with the Kolmogorov-Kraichnan theory.

An important feature of 2D turbulence is the presence of the inverse energy cascade, or the transfer of energy from smaller to larger scales in the inertial range $r_{f}<r<r_{\text {low }}$. A direct way to prove the existence of the inverse energy cascade is to compute the third moment of the velocity increments across a distance $r$ in a flow, $\delta V(r)=V(l+r)-V(l)$. The third order moment $S_{3 L}=<\left[\delta V_{L}(r)\right]^{3}>$ (where index $L$ refers to the longitudinal velocity component with respect to $\boldsymbol{r}$, and angular brackets denote averaging over all possible positions $l$ within the flow field, and in time, over many realizations) is related to the spectral energy flux $\varepsilon$ via the Kolmogorov flux relation. In the inertial range of 2D turbulence this relation reads as ${ }^{10,33}$

$$
S_{3 L}=\frac{3}{2} \varepsilon r .
$$



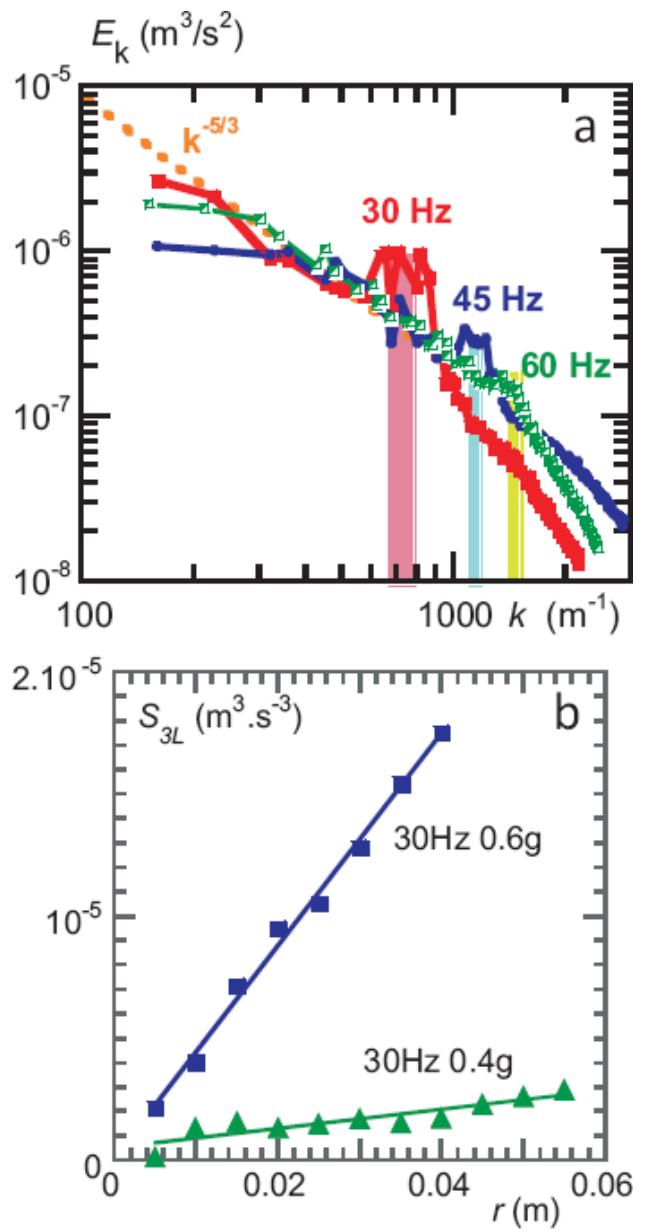

Fig. 5. (a) Energy spectrum in the surface wave driven turbulence at different frequencies $(30 \mathrm{~Hz}, 45 \mathrm{~Hz}, 60 \mathrm{~Hz}$ ), and at similar supercriticalities. Colored vertical bars show the forcing ranges. (b) Third moment of the longitudinal velocity increments measured at $f_{0}=30 \mathrm{~Hz}$ at different supercriticalities $\alpha=1(a=0.4 g)$, and $\alpha=2(a=0.6 g)$.

Fig. 5(b) shows third order structure functions computed from the velocity fields measured in the surface wave driven turbulence at $f_{0}=30 \mathrm{~Hz}$ for two different vertical accelerations. In both cases $S_{3 L}$ is a positive linear function of $r$. The spectral energy flux $\varepsilon$ can be computed from Eq. (6). The derived values are in agreement with the spectral power densities derived from the spectra of Fig. 5(a).

The existence of the inverse energy cascade in a bounded flow is a prerequisite for the accumulation of the turbulence energy at the scale of the boundary size ${ }^{2}$. But the spectral condensation, or the generation of large coherent structures, is dependent on the boundary size, the energy flux, or the damping rate. It has been demonstrated in experiments in electromagnetically driven turbulence that in a square box, whose size is 
smaller than the dissipation scale, turbulent inverse energy cascade can drive large scale vortices which are coherent over the entire domain ${ }^{10}$. To test if the inverse energy cascade can generate coherent vortices in the surface wave driven turbulence, we produce Faraday flows in a square container $110 \times 110 \mathrm{~mm}^{2}$ at the excitation frequency of $f_{0} \approx 30$ $\mathrm{Hz}$, which gives the forcing scale of $l_{f} \approx 7.7 \mathrm{~mm}$. Fig. 6(a) shows trajectories of particles in this smaller square container. A large vortex occupying the entire box develops in the square container about 60 seconds after switching on the shaker. Total kinetic energy of the flow stabilizes after this time marking a steady state in which energy flux delivered via the inverse cascade is balanced by viscous dissipation and the wall drag. The kinetic energy spectrum of spectrally condensed flow is substantially steeper than the Kolmogorov spectra in Fig. 4(b), $E(k) \propto k^{-4}$, as shown in Fig. 6(b), similarly to the observations in electromagnetically driven turbulence ${ }^{10}$.

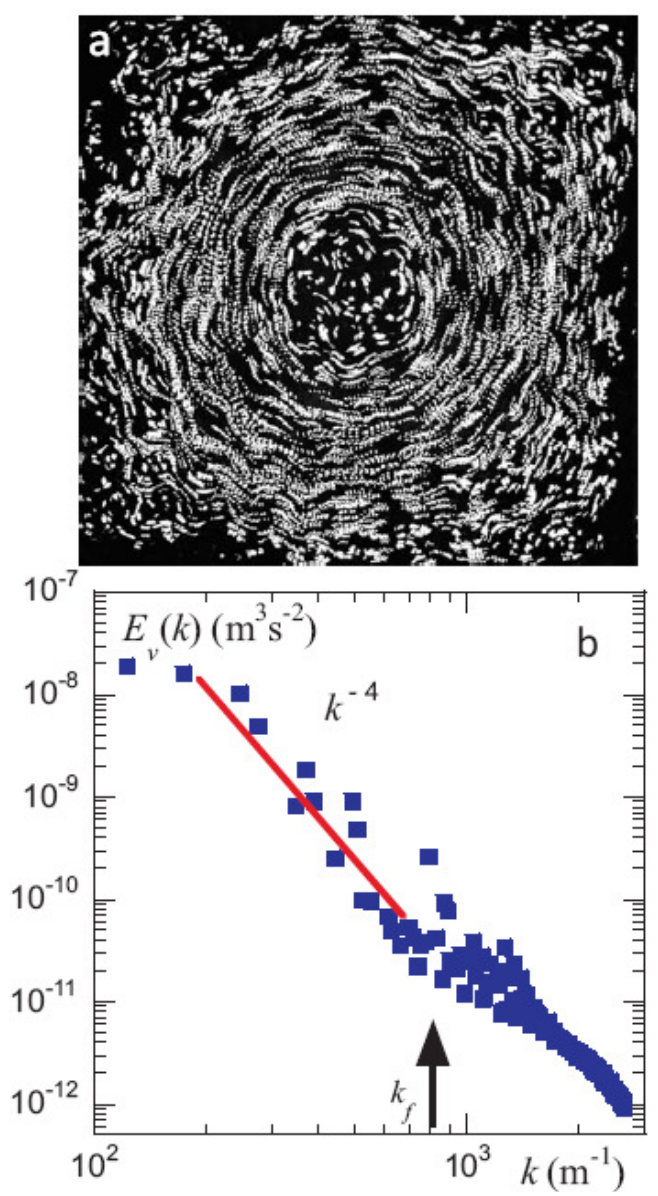

Fig. 6. (a) Particles trajectories followed over 4 periods of the shaker oscillations in a spectrally condensed flow in a square box. The large scale vortex is $10 \mathrm{~cm}$ in diameter. The particles diameter is 50 microns. (b) Kinetic energy spectrum in the spectrally condensed turbulence. 


\section{Discussion and Conclusions}

We have shown that the motion of floaters on the surface of the water perturbed by the Faraday waves shows many similarities with the motion of fluid parcels in 2D turbulence. Such a motion can be suppressed by, for example, placing thin film of bovine serum, as has been shown in Ref. 23. Such a film would introduce rather strong dissipation and would stop fluid particles on the surface from moving horizontally. If however such dissipation is removed (e.g. by adding surfactant), floaters execute chaotic motion. This coincides with disordering of the Faraday wave field, sometime interpreted as the generation of surface wave turbulence.

More studies are needed to clarify the detail of the 3D motion of floaters on the Faraday ripples. In particular, it is of interest whether particle trajectories show signatures of diffusion and of Levy flights, as it has been found in rotating flows ${ }^{34}$. This may also clarify why energy injected into the particles' horizontal motion remains localized in $k$-space and can thus support inertial interval in 2D turbulence.

To conclude, experimental results support the existence of the inverse energy cascade fueled by Faraday waves. Faraday flows represent thus a new versatile tool of laboratory modeling of $2 \mathrm{D}$ turbulence. It is remarkable that the inverse energy cascade, initially thought of as a process intrinsic only to idealized 2D turbulence is found in a variety of physical systems where its existence could not possibly be expected a priori5' 6 ' 8 . In 3D turbulence, the inverse energy cascade was recently found to naturally coexist with the direct energy cascade ${ }^{11}$.

\section{Acknowledgments}

This work was supported by the Australian Research Council's Discovery Projects funding scheme (DP110101525). HX would like to acknowledge the support by the Australian Research Council's Discovery Early Career Research Award (DE120100364).

\section{References}

1. U. Frisch, Turbulence: The legacy of A. N. Kolmogorov, (Cambridge Univ. Press, 1995).

2. R.Kraichnan, Phys. Fluids 10, 1417 (1967).

3. J. Sommeria, J. Fluid Mech. 170, 139 (1986).

4. J. Paret and P. Tabeling, Phys. Rev. Lett. 79, 4162 (1997).

5. D. Byrne, H. Xia, and M. Shats, Phys. Fluids 23, 095109 (2011).

6. H. Xia, D. Byrne, G. Falkovich, and M. Shats, Nature Physics 7, 321 (2011).

7. D. Byrne, and J.A. Zhang, Geophys. Res. Lett. 40, 1439 (2013).

8. A. von Kameke, F. Huhn, G. Fernández-García, A.P. Muñuzuri, and V. Pérez-Muñuzuri, Phys. Rev. Lett. 107, 074502 (2011).

9. N. Francois, H. Xia, H. Punzmann, and M. Shats Phys. Rev. Lett. 110, 194501 (2013).

10. H. Xia, M. Shats and G. Falkovich, Phys. Fluids 21, 125101 (2009).

11. L. Biferale, S. Musacchio, and F. Toschi, Phys. Rev. Lett. 108, 164501 (2012).

12. A. Kudrolli and J.P. Gollub, Physica D 97, 133 (1996).

13. D.I. Goldman, M.D. Shattuck, Sung Joon Moon, J.B. Swift and H.L. Swinney, Phys. $\square$ Rev. Lett. 90, 104302 (2003). 
14. A.V. Kityk, J. Embs, V.V. Mekhonoshin and C. Wagner, Phys. Rev. E 72, 036209 (2005).

15. I. Shani, G. Cohen, and J. Fineberg, Phys. Rev. Lett. 104, 184507 (2010).

16. W. B. Wright, R. Budakian, and S.J. Putterman, Phys. Rev. Lett. 76, 4528 (1996).

17. D. Snouck, M.-T. Westra, and W. van de Water, Phys. Fluids 21, 025102 (2009).

18. E. Falcon, Discr. Contin. Dynam. Sys. - Series B, 13, 819 (2010).

19. J. Wu, R. Keolian, and I. Rudnick, Phys. Rev. Lett. 52, 1421 (1984).

20. O. Lioubashevski, H. Arbell and J. Fineberg, Phys. Rev. Lett. 76, 3959 (1996).

21. H. Xia, M. Shats, and H. Punzmann, EPL 91, 14002 (2010).

22. J. Rajchenbach, A. Leroux, and D. Clamond, Phys. Rev. Lett. 107, 024502 (2011).

23. M. Shats, H. Xia, and H. Punzmann, Phys. Rev. Lett. 108, 034502 (2012).

24. Y. Couder, S. Protiere, E. Fort, and A. Boudaoud, Nature 437, 208 (2005).

25. M. Shats, H. Punzmann, and H. Xia, Phys. Rev. Lett. 104, 104503 (2010).

26. M. Faraday, Philos. Trans. Royal Soc. of London 121, 319 (1831).

27. L. F. McGoldrick, J. Fluid Mech. 52, 725 (1965).

28. T. B. Benjamin, and J.E. Feir, J. Fluid Mech. 27, 417 (1967).

29. G. D. Crapper, J. Fluid Mech. 2, 532 (1957).

30. H. Punzmann, H. Xia, and M. Shats, Phys. Rev. Lett. 103, 064502 (2009).

31. H. Xia, M. Shats, and H. Punzmann, EPL 91, 14002 (2010).

32. H. Xia, T. Maimbourg, H. Punzmann, and M. Shats, Phys. Rev. Lett. 109, 114502 (2012).

33. V. Yakhot, Phys. Rev. E 60, 5544 (1999).

34. T. H. Solomon, E.R. Weeks, and H. L. Swinney, Physica D 76, 70 (1994). 